\title{
A COMPARISON OF SELECTED PATTERN RECOGNITION FUNCTIONS*
}

\author{
William A. Porter \\ Department of Computer, Information, and Control Engineering. \\ The University of Michigan. Ann Arbor. MI 48109, U.S.A.
}

(Received 2 September 1976)

\begin{abstract}
In this study computer simulation is used to compare selected pattern recognition functions. The Highleyman deck of 50 hand written characters provides one comparative data base. A second data base is derived from multispectral infrared sensor data taken over California's Imperial Valley. Emphasis is placed on comparing the classical minimum distance recognition functions with two new recognition functions introduced in a recent predecessor article.
\end{abstract}

Pattern recognition Geometric clustering Computer simulation Comparative performance Multispectral processing handwritten character recognition.

\section{INTRODUCTION}

Suppose that $X$ denotes a universe of patterns and that $\Gamma_{i} \subset X, i=1, \ldots, m$ is a disjoint cover of $X$, that is $\Gamma_{i} \cap \Gamma_{j}=\phi, i \neq j$ and $X=\cup_{i} \Gamma_{i}$. If $Y=\left\{y_{1} \ldots y_{m}\right\}$, is a distinct (signature) set then a function $F: X \rightarrow Y$ is said to recognize the patterns $\left\{\Gamma_{i}\right\}$ if

$$
F(x)=y_{i} \quad \text { all } x \in \Gamma_{i} \quad i=1, \ldots, m .
$$

In most applications such an idealized definition of a pattern recognition function is unworkable. A sensible modification which often results is that by an averaging, or expectation of clustering process a typifying set of points $x_{i} \in \Gamma_{i} i=1, \ldots, m$, is chosen. The function $F$ is asked to satisfy $F\left(x_{i}\right)=y_{i}$ $i=1, \ldots, m$. Moreover, it is hoped that, in some metric, for $x \in \Gamma_{i}, F(x)$ is closer to $y_{i}$ than to $y_{j} j \neq i$. This latter description is compatible with statistical as well as geometric interpretations and can be viewed as a function sensitivity problem.

The recognition functions considered in this study include the well known minimum distance function and its related maximum probability function for Gaussian error. Comparison is made with two recognition functions ${ }^{(2)}$; namely, the linear function constructed using dual sets and a polynomic function which in some ways resembles a committee machine.

The results reported here were obtained both by computer simulation and by testing on standard data bases. The computer simulation which is detailed later, utilized a random number data base with variable standard deviation. In a second test the Highleyman deck ${ }^{(1)}$ of fifty hand written characters was utilized. Finally, multispectral data, taken over California's Imperial Valley, was utilized with the $\Gamma_{i}$ consisting of the emmissions from the various crops present.

* Sponsored in part by the Air Force Office of Scientific Research under Grant AFOSR 73-2427 and 77-0352.

\section{PRELIMINARIES}

It is noted ${ }^{(2)}$ that Hilbert spaces provide a natural setting for studies of the pattern recognition problem. In the simulation studies a concrete Hilbert space. namely $R^{n}$ equipped with the natural innerproduct

$$
\langle x, y\rangle=\sum_{j=1}^{n} x^{j} y^{j}, \quad x, y \in R^{n},
$$

was used. The norm on $R^{n}$ is the usual one namely $\|x\|^{2}=\langle x, x\rangle, x \in R^{n}$.

The Highleyman character deck consists of fifty handwritten samples of the twenty-six alphabetic characters plus the ten integers $0, \ldots, 9$. Each character was scanned on a $12 \times 12$ lattice which encoded each character as a 144 binary tuple. Thus $n=144$ in the above inner product while each $\Gamma_{i}, i=1, \ldots, 36$ consists of the fifty coded versions of a single character. The typifying element $\bar{\gamma}_{i} \in \Gamma_{i}$ was taken to be the average tuplet.

$$
\bar{\gamma}_{i}=\frac{1}{50} \sum_{\Gamma_{i}} \gamma_{j}, \quad i=1, \ldots, 36
$$

While each coded character has binary entries the averages, $\bar{\gamma}_{i}$. do not and in general have components $0 \leq \bar{\gamma}_{i}^{j} \leq 1$, taking on values in multiples of $1 / 50$. In the same vein the deviations $\gamma_{i}-\bar{\gamma}_{i}$ for $\gamma_{i} \in \Gamma_{i}$ about the average are tuplets with both negative and positive entries.

The Highleyman data base can be preprocessed in several ways. Having computed the $\bar{\gamma}_{i}, i=1, \ldots, 36$ one can compute the covariance matrices

$$
\Phi_{i}=\frac{1}{49} \sum_{\Gamma_{1}}\left(\gamma-\gamma_{i}\right)\left(\gamma-\bar{\gamma}_{i}\right)^{t}, \quad i=1, \ldots, 36,
$$

and construct an innerproduct(s)

$$
\langle x, y\rangle_{\Phi}=\left\langle x, \Phi^{-1} y\right\rangle,
$$

which would reflect a Gaussian assumption on the deviations $\left(\eta_{i}-\bar{\gamma}_{i}\right)$. Secondly, the identification 
algorithm could be allowed to shift the individual patterns as a means of improving an appropriate measure of confidence in the identification. Other authors $^{(1,3)}$ have reported marked improvement when pattern shifting is allowed.

In our experiments the presence of substantial pattern variation is actually helpful in documenting the properties of the identification functions. For the Highleyman data base, the numbers

$$
\Delta_{i}=\frac{1}{50} \sum_{\gamma \in \Gamma_{i}}\left\|\gamma-\bar{\gamma}_{i}\right\| \quad i=1, \ldots, 36,
$$

were computed and it was found that the errors were often bigger than the patterns in that $\Delta_{i}$ ranged as large as $125 \%$ of $\left\|\bar{\gamma}_{i}\right\|$. It was also noted that the $\Delta_{i}$ were roughly proportionate to the $\left\|\vec{\gamma}_{i}\right\|$. This is intuitive since the misshape of a letter with many unit entries produces an error with many unit entries in the respective code vectors. To illustrate, the numerical values of $\left\|\bar{\gamma}_{i}\right\|$ and $\Delta_{i}$ are listed below for the ten numerals $1, \ldots, 9,0$.

The covariance matrices $\Phi_{i}$ defined earlier were also computed for selected $\Gamma_{i}$ and found to have substantial nondiagonal entries. To avoid needless complexity the simulation utilized an error data base constructed from a random number generator with the Highleyman data base used as a consistency check at the end of the simulation.

The error data base consisted of 300 tuplets of length 144 drawn from a Gaussian random number generator. The data base was given minor adjustments so that the finite sample average and the finite sample standard deviation for each vector component were zero and one respectively.

\section{THE RECOGNITION FUNCTIONS}

In this section we summarize the definitions and properties of the various recognition functions that were tested in the simulation.

\section{The dual functions}

Consider once more the thirty-six pattern classes $\Gamma_{i}$ of the Highleyman data bank and the associated mean patterns $\hat{\gamma}_{i}$ computed in equation (1). Each $\bar{\gamma}_{i}$ is a 144 tuple with components $0 \leq \bar{\gamma}_{i}^{j} \leq 1$. It was found that the set $\left\{\bar{y}_{i}\right\}$ is linearly independent in $R^{n}$ with $n=144$. Thus the dual set $\bar{y}_{i}^{+}$satisfying

Linear span $\left\{\bar{\gamma}_{i}\right\}=$ linear span $\left\{\bar{\gamma}_{i}^{+}\right\}$

$$
\left\langle\bar{\gamma}_{i}, \gamma_{j}^{+}\right\rangle=\delta_{i j} \quad i, j=1, \ldots, 36
$$

can be readily determined ${ }^{(2)}$.

The dual set has properties relevant to pattern recognition. First, note that the $\left\langle\bar{\gamma}_{i}^{+}, \hat{\gamma}_{j}\right\rangle=\delta_{i j}$ property means that the functions $\left\{\phi_{i}\right\}$ defined by

$$
\phi_{i}(\gamma)=\left\langle\bar{\gamma}_{i}^{+}, \gamma\right\rangle \quad i=1, \ldots, 36,
$$

qualify as recognition functions.

Secondly, we note that the matrix

$$
P=\sum_{1}^{36} \bar{\gamma}_{i}\left(\bar{\gamma}_{i}^{+}\right)^{t}
$$

is readily computable and is, in fact, the orthogonal decomposition of $R^{n}$ onto the linear span of $\left\{\bar{\gamma}_{i}\right\}$. Thus if $\gamma \in R^{n}$ is arbitrary $P \gamma$ is the closest element to $\gamma$ in span $\left\{\bar{\gamma}_{i}\right\}$. Moreover, $P \gamma$ is a linear combination

$$
P \gamma=\sum_{1}^{36} \alpha_{i} \bar{\gamma}_{i}
$$

where

$$
\alpha_{i}=\phi_{i}(P \gamma)=\phi_{i}(\gamma) \quad i=1, \ldots, 36 .
$$

In general then for arbitrary

$$
\gamma=\sum_{1}^{36} \bar{\gamma}_{i} \phi_{i}(\gamma)+(I-P) \gamma .
$$

The ratio $\|P \gamma\| /\|\gamma\|$ can be viewed as a noise suppression factor.

In the simulation the recognition function constructed by choosing the maximum of the numbers $\left\{\phi_{i}(\gamma): i=1, \ldots, 36\right\}$ was tested. This function, which is referred to as linear case 1 , chooses the maximum coefficient in the best fit linear expansion of $\gamma$ in the subspace span $\left\{\bar{\gamma}_{i}\right\}$.

\section{Corollation technique}

In many early studies character recognition schemes of the following type were considered. Define the functions

$$
\pi_{i}(\gamma)=\left\langle\bar{\gamma}_{i}, \gamma\right\rangle \quad i=1, \ldots, 36,
$$

and choose $i$ such that $\pi_{i}(\gamma) \geq \pi_{j}(\gamma)$ all $j \neq i$. A variation on this used the normalized function defined by

$$
\bar{\pi}_{i}(\gamma)=\left\langle\bar{\gamma}_{i}, \gamma\right\rangle / \mid \bar{\gamma}_{i}\|\cdot\| \gamma \| \quad i=1, \ldots, 36,
$$

which is interpretable as the direction cosine set resolving $\gamma$ along the $\left\{\bar{\gamma}_{i}\right\}$.

When the set $\left\{\bar{\gamma}_{i}\right\}$ has several almost colinear subsets, that is $\left\langle\bar{\gamma}_{i}, \bar{\gamma}_{j}\right\rangle \simeq\left\|\bar{\gamma}_{i}\right\| \cdot \| \bar{\gamma}_{j}||$, it has been suggested that penalty weights be added to the $\bar{\gamma}_{i}$ to more or less help orthogonalize the test pattern set. The ultimate of this, however, is to use the dual set described above, and we shall not mention penalty methods further.

Of more importance is the relationship of the $\pi_{i}$ to statistical estimation. Assuming that the class $\Gamma_{k}$ is Gaussian distributed with mean $\bar{\gamma}_{k}$ and covariance

Table 1. Pattern and pattern deviation measures

\begin{tabular}{lcccccccccc}
\hline$\Gamma_{i}$ & $\{1\}$ & $\{2\}$ & $\{3\}$ & $\{4\}$ & $\{5\}$ & $\{6\}$ & $\{7\}$ & $\{8\}$ & $\{9\}$ & $\{0\}$ \\
\hline$\left\|\bar{\gamma}_{i}\right\|$ & 2.46 & 3.17 & 3.18 & 3.62 & 3.14 & 3.39 & 3.22 & 3.78 & 3.62 & 3.20 \\
$\Delta_{i}$ & 2.42 & 3.67 & 3.72 & 3.59 & 3.86 & 3.71 & 3.23 & 4.09 & 3.71 & 3.91 \\
\hline
\end{tabular}


matrix $\Phi_{k}$ it is well known ${ }^{(4)}$ that the relevant conditional distribution function is given by

$$
P\left(\gamma / \Gamma_{k}\right)=\lambda_{k} \exp \left\{-\frac{1}{2}\left\|\gamma-\bar{\gamma}_{k}\right\|_{k}^{2}\right\}
$$

where:

$$
\left(\lambda_{k}\right)^{-2}=(2 \pi)^{n} \operatorname{det} \Phi_{k}
$$

and

$$
\left\|\gamma-\bar{\gamma}_{k}\right\|_{k}=\left\langle\gamma-\bar{\gamma}_{k}, \Phi_{k}^{-1}\left(\gamma-\bar{\gamma}_{k}\right)\right\rangle .
$$

When $\Phi_{k}=I, k=1, \ldots, 36$ and noting the monotonicity of the exponential function we are led naturally to consider the functions

$$
p_{k}(\gamma)=\| \gamma^{\prime}-\left.\bar{\gamma}_{k}\right|^{2} . \quad k=1, \ldots, 36 .
$$

Using the orthogonal projection, $P$, defined earlier we have

$$
P_{k}(\gamma)=\left\|(I-P)_{\gamma}\right\|^{2}+\left\|P_{\gamma}-\bar{\gamma}_{k}\right\|^{2}, \quad k=1, \ldots, 36 .
$$

Since $\|(I-P)\|^{2}$ is constant in all functions we delete the term and focus on

$$
\bar{p}_{k}(\gamma)=\left\|P \gamma-\bar{\gamma}_{k}\right\|^{2} . \quad k=1, \ldots, 36 .
$$

Similarly we note that

$$
\left|P \gamma_{\gamma}-\bar{\gamma}_{k}{ }^{2}=\left\|P \gamma_{\gamma}\right\|^{2}-2\left\langle\gamma_{i} \bar{\gamma}_{k}\right\rangle+\| \bar{\gamma}_{k}\right|^{2} .
$$

and hence the functions

$$
q_{k}(\gamma)=-2 \pi_{k}(\gamma)+\left\|\bar{\gamma}_{k}\right\|^{2},
$$

give the same ordering as $p_{k}$ and $\bar{p}_{k}$.

In the simulation the recognition function formed by choosing the minimum of the set of $\left\{\bar{p}_{k}(\gamma)\right.$ $k=1, \ldots, 36\}$ was studied. This case, which is referred to as linear case 2, was implemented using equation (6).

\section{The polynomic functions}

Our interest here is with a specific class of polynomic functions introduced in Ref. 2. These functions are defined by

$$
\psi_{k}(\eta)=\prod_{j \neq k}\left\{\frac{\left\langle\gamma-\bar{\gamma}_{j}, \bar{\gamma}_{k}-\bar{\gamma}_{j}\right\rangle}{\mid \bar{\gamma}_{k}-\bar{\gamma}_{j} \|^{2}}\right\}, \quad k=1, \ldots, 36 .
$$

As with the dual functions of equation (4), we have

$$
\psi_{k}\left(\gamma_{j}\right)=\delta_{j k}, \quad j, k=1, \ldots, 36
$$

We note. however, that the $\left\{\psi_{k}\right\}$ are well defined provided only that the set $\left\{\bar{\gamma}_{j}\right\}$ is distinct. It is also easily seen that each $\psi_{k}$ is a polynomic operator of order thirty-five. This sets it apart from other polynomic recognition functions considered in the literature.
All of the recognition functions defined earlier utilized explicitly or implicitly the $\left\{\bar{\gamma}_{j}\right\}$. The polynomic function, of course, utilizes the differences $\left\{\bar{\gamma} j-\overline{\gamma_{i}}\right\}$. This results in a distinctive behavior when, for instance, $\bar{\gamma}_{1}, \bar{\gamma}_{2}$, and $\bar{\gamma}_{3}$ are almost colinear but have differences $\bar{\gamma}_{1}-\bar{\gamma}_{2}, \bar{\gamma}_{2}-\bar{\gamma}_{3}, \bar{\gamma}_{3}-\bar{\gamma}_{4}$ which are nearly orthogonal.

\section{THE SIMULATION TESTS}

The statistical simulations summarized in this section were developed for several reasons. First, it was found that simulations on the entire thirty-six symbol Highleyman data base were consistent with simulations on the ten pattern subset consisting of the integers. As a result. attention was focused on the integers with suitable simulations on the larger data base to check for consistency. The net effect was a considerable savings in computer time and cost.

As to the statistical properties of the Highleyman data sets $\Gamma_{i}$, related to the integers, some of these are summarized in the following table where

$$
\begin{array}{rlrl}
\bar{n} & =\frac{1}{50} \sum_{1}^{50} n_{i} & n & =0,1 \ldots, 9 \\
\Delta_{n} & =\frac{1}{50} \sum_{1}^{50}\left|n_{i}-\bar{n}\right| & n & =0,1 \ldots, 9 \\
v_{n} & =\left[\frac{1}{49} \sum_{1}^{50}|| n_{i}-\left.\bar{n}_{i}\right|^{2}\right]^{1 / 2} & \\
n & =0,1 \ldots, 9
\end{array}
$$

$\left\{\bar{n}^{+}\right\}$is the dual of $\{\bar{n}\}$, and $\sigma_{n}=\left[v_{n}^{2}-\Delta_{n}^{2}\right]^{12}$.

Concerning these statistical properties, we note that in each case $\Delta_{n}$ and $v_{n}$ are approximately the same size as $\|\bar{n}\|$. This indicates the fluctuations about the mean pattern are often as big as the mean pattern itself.

As a prelude to the experiment using the Highleyman data base simulations were run using a Gaussian error data base. The purpose of these simulations was to help isolate properties related to the pattern recognition functions from properties related to the possibly non-Gaussian characteristic of the Highleyman data base.

In the next two tables the properties of the polynomic functions of equation (8) and the linear functions of equation (4) are compared. In both simulations the input set was compiled by choosing $m \in\{\overline{0}, \overline{2} . \overline{4}, \overline{6}, \overline{8} ;$ and then forming $\mu_{i}=m+k v_{i}$ where $v_{i} \in \boldsymbol{V}$ and $k /\|m\|$ was chosen in multiples of 0.2 as indicated on the Tables.

Table 2. Data base variation measures

\begin{tabular}{lcccccccccc}
\hline \multicolumn{1}{c}{$\mathrm{n}$} & 1 & 2 & 3 & 4 & 5 & 6 & 7 & 8 & 9 & 0 \\
\hline$\|\bar{n}\|$ & 2.46 & 3.17 & 3.18 & 3.62 & 3.14 & 3.39 & 3.22 & 3.78 & 3.62 & 3.20 \\
$\left\|n^{+}\right\|$ & 0.76 & 0.87 & 0.89 & 0.97 & 0.83 & 0.79 & 0.76 & 1.08 & 1.08 & 0.68 \\
$\Delta_{n}$ & 2.42 & 3.67 & 3.72 & 3.59 & 3.86 & 3.71 & 3.23 & 4.09 & 3.71 & 3.91 \\
$v_{n}$ & 2.48 & 3.74 & 3.79 & 3.65 & 3.94 & 3.81 & 3.30 & 4.20 & 3.80 & 3.99 \\
$\sigma_{n}$ & 0.61 & 0.72 & 0.72 & 0.65 & 0.79 & 0.85 & 0.69 & 0.96 & 0.83 & 0.78 \\
\hline
\end{tabular}


Table 3. $M\left[\psi_{4}(m, k)\right]$ and $\sigma\left[\psi_{4}(m, k)\right]$ respectively

\begin{tabular}{cccccc}
\hline$m$ & $\overline{2}$ & $\overline{4}$ & $\overline{6}$ & $\overline{8}$ & $\overline{0}$ \\
\hline $\begin{array}{c}m \\
k /\|m\|\end{array}$ & $0.00(0.00)$ & $1.00(0.00)$ & $0.00(0.00)$ & $0.00(0.00)$ & $0.00(0.00)$ \\
0.0 & $0.00(0.00)$ & $1.02(0.21)$ & $0.00(0.00)$ & $0.00(0.00)$ & $0.00(0.00)$ \\
0.2 & $0.00(0.00)$ & $1.06(0.45)$ & $0.00(0.00)$ & $0.00(0.00)$ & $0.00(0.00)$ \\
0.4 & $0.00(0.00)$ & $1.14(0.74)$ & $0.00(0.00)$ & $0.00(0.00)$ & $0.00(0.00)$ \\
0.6 & $0.00(0.00)$ & $1.26(1.06)$ & $0.00(0.01)$ & $0.00(0.01)$ & $0.00(0.00)$ \\
0.8 & $0.00(0.00)$ & $1.42(1.56)$ & $0.00(0.02)$ & $0.00(0.01)$ & $0.00(0.00)$ \\
1.0 & $0.00(0.00)$ & $1.64(2.21)$ & $0.01(0.03)$ & $0.01(0.03)$ & $0.00(0.01)$ \\
1.2 & & &
\end{tabular}

For convenience the notation

$$
\begin{aligned}
M\left[\psi_{4}(m, k)\right]= & \frac{1}{300} \sum_{1}^{300} \psi_{4}\left(m+k v_{i}\right) \\
\sigma\left[\psi_{4}(m, k)\right]= & \left\{\frac { 1 } { 2 9 9 } \sum _ { 1 } ^ { 3 0 0 } \left[\psi_{4}\left(m+k v_{i}\right)\right.\right. \\
& \left.\left.-M\left[\psi_{4}(m, k)\right]\right]^{2}\right\}^{1 / 2},
\end{aligned}
$$

is adopted. The quantities $M\left[\varphi_{4}(m, k)\right]$ and $\sigma\left[\varphi_{4}(m, k)\right]$ are defined analogously, however, the linearity of the $\varphi_{i}$ and the unbiased nature of $v$ simplifies these latter computations. In the following tables the values of $\sigma\left[\psi_{4}(m, k)\right]$ and $\sigma\left[\varphi_{4}(m, k)\right]$ are placed in parentheses.

The most interesting property displayed in these two tables is the ability of $\psi_{4}$ to reject false patterns. For Gaussian distribution errors with standard deviations as large as $120 \%$, of the input pattern, the function $\psi_{4}$ continues to reject false patterns, that is $m \in\{\overline{2}, \overline{6}, \overline{8}, \overline{0}\}$, with remarkable consistency. The correct pattern, $m=\overline{4}$, is recognized by $\psi_{4}$ with a confidence level which varies inversely with error size. For $k /\|m\|=0.9$ the sample $\left\{\psi_{4}\left(\overline{4}+k v_{i}\right)\right\}$ mean and S.D. are approximately the same and hence occasional small (or even negative) numbers appear. The tenacity with which $\psi_{4}$ rejects the false patterns suggests the misclassification errors can be easily minimized by imposing a threshold level, $\mu$. If the maximum $\psi_{j}(u)$ does not exceed $\mu$ then the pattern is not classified.

Before leaving Table 4 it is noted that simulations of the $\psi_{2}, \psi_{6}, \psi_{8}$ and $\psi_{0}$ on the same input set produced similar behavior. The direct use of the Highleyman data base also confirms the properties of the $\psi$ functions summarized here.

As a footnote to the above data on the functions $\left\{\varphi_{k}\right\}$ we recall that the operator, $P$, of equation (5) is linear. When $P$ projects on the linear subspace spanned by $\{\overline{0}, \overline{1}, \ldots, \overline{9}\}$ it reduces dimensions from 144 to 10 . Thus, on the average, $\left\|P v_{i}\right\| /\left\|v_{i}\right\| \simeq 1 / \sqrt{14.4}$. By experiment it was determined that for the data base used

$$
\frac{1}{300} \sum_{v_{i} \in V}\left\|P v_{i}\right\| /\left\|v_{i}\right\|=0.26=1 / \sqrt{14.8}
$$

Turning now to the Highleyman data base, the polynomic character recognition functions were applied to the entire data base. The results summarized in the following tables are entirely indicative of the total simulation.

In Table 5 the outputs of $\left\{\psi_{i}: i=1, \ldots, 9,0\right\}$ are recorded when the 50 copies of the numeral 4 are applied. In examining these data it is clear that the functions $\psi_{1}$ for $i=1,2,3,5,7$ output $\simeq 0$ for all inputs. The functions $\psi_{i}$ for $i=6,8,0$ output numbers $>0.20$ a total of 4 times. The function $\psi_{9}$ is more active giving a $>0.20$ reading 10 times. The function $\psi_{4}$ gives outputs which vary widely. The output of $\psi_{4}$ fails to be maximum among the 10 outputs a total of 10 times. This only happens twice, however, when the $\psi_{4}$ output is $>0.20$.

In Table 6 the function $\psi_{4}$ is applied to each of the 50 copies of the 10 numerals. We note that $\psi_{4}$ never responds to numerals $1,3,5$; to numerals $2,6,7,8,0$ it gives a total of 12 readings $>0.20$ and for 9 gives outputs $>0.20$ a total of 13 times. Although the data displayed is different, Tables 3,5 and 6 all have a similar character.

For comparison purposes the experiments summarized in Tables 5 and 6 were repeated using the $\varphi_{i}$ functions of equation (4). The contrast between Tables 5 and 7 and Tables 6 and 8 is visually apparent and in the linear cases an error is picked up in all recognition functions. In the polynomic case errors

Table 4. $M\left[\varphi_{4}(m, k)\right]$ and $\sigma\left[\varphi_{4}(m, k)\right]$ respectively

\begin{tabular}{cccccc}
\hline$m$ & $\overline{2}$ & $\overline{4}$ & $\overline{6}$ & $\overline{8}$ & $\overline{0}$ \\
$k /\|m\|$ & & & & & \\
0.0 & $0.00(0.00)$ & $1.0(0.00)$ & $0.00(0.00)$ & $0.00(0.00)$ & $0.00(0.00)$ \\
0.2 & $0.00(0.05)$ & $1.0(0.06)$ & $0.00(0.05)$ & $0.00(0.06)$ & $0.00(0.05)$ \\
0.4 & $0.00(0.10$ & $1.0(0.12)$ & $0.00(0.11)$ & $0.00(0.13)$ & $0.00(0.11)$ \\
0.6 & $0.00(0.15)$ & $1.0(0.18)$ & $0.00(0.17)$ & $0.00(0.19)$ & $0.00(0.16)$ \\
0.8 & $0.00(0.21)$ & $1.0(0.24)$ & $0.00(0.23)$ & $0.00(0.25)$ & $0.00(0.21)$ \\
1.0 & $0.00(0.26)$ & $1.0(0.30)$ & $0.00(0.28)$ & $0.00(0.31)$ & $0.00(0.26)$ \\
1.2 & $0.00(0.32)$ & $1.0(0.36)$ & $0.00(0.34)$ & $0.00(0.35)$ & $0.00(0.32)$ \\
\hline
\end{tabular}


have an effect in at most two or three of the recognition functions.

For completeness of our comparison, the classic maximum probability recognition functions were also tested. This was done indirectly by using the related functions $\bar{\rho}_{k}$ of equation (6) in the same experiment. The results are given in Tables 9 and 10. Noting that the smallest number is the maximum probable choice, it is easily determined that Tables 9,10 have the same character as Tables 7,8 respectively.

In numerical terms Table 5 has 9 false maximums, Table 7 has 15 false maximums and Table 9 has 19 false minimums On a much larger simulation, it was found that polynomic recognition function excelled over the minimum distance function which excelled over the linear recognition function. The margins of gain varied, but were generally closer than that of the data of Tables 5, 7, and 9 .

\section{CLOSING}

In addition to the experiments described previously, some preliminary experiments were run using multispectral data taken over California's Imperial Valley. The results of these experiments have not been included here because of the limited size of the data sample available and the relatively sparse documentation of these data. The experiments run, however, did not conflict with the Tables of the previous Section.

Table 5. The $\psi_{i}$ responses to the numeral 4

\begin{tabular}{|c|c|c|c|c|c|c|c|c|c|}
\hline$\psi_{1}$ & $\psi_{2}$ & $\psi_{3}$ & $\psi_{4}$ & $\psi_{5}$ & $\psi_{6}$ & $\psi_{7}$ & $\psi_{8}$ & $\psi_{9}$ & $\psi_{0}$ \\
\hline-0.00 & 0.00 & 0.00 & 1.60 & 0.00 & -0.00 & -0.00 & 0.72 & 1.31 & -0.00 \\
\hline 0.00 & 0.00 & 0.00 & 0.44 & 0.00 & 0.01 & 0.00 & 0.00 & 0.01 & -0.00 \\
\hline-0.00 & -0.00 & 0.00 & 1.09 & 0.04 & 0.00 & -0.00 & -0.00 & 0.08 & 0.00 \\
\hline 0.00 & 0.00 & 0.02 & 0.02 & 0.00 & 0.00 & 0.00 & -0.00 & 0.07 & 0.00 \\
\hline 0.00 & 0.00 & 0.00 & 0.49 & 0.00 & -0.00 & 0.07 & 0.00 & 0.07 & 0.00 \\
\hline 0.00 & -0.00 & 0.00 & 0.33 & 0.00 & -0.00 & 0.00 & 0.03 & 0.26 & 0.00 \\
\hline 0.00 & 0.00 & 0.00 & 0.08 & 0.01 & 0.00 & 0.03 & -0.00 & 0.02 & 0.00 \\
\hline 0.00 & 0.00 & 0.00 & 16.56 & -0.00 & -0.00 & 0.00 & 0.01 & -0.67 & 0.00 \\
\hline-0.00 & 0.09 & 0.00 & 0.08 & -0.00 & 0.14 & 0.00 & 0.00 & -0.00 & 0.00 \\
\hline-0.00 & 0.02 & 0.00 & 0.75 & -0.00 & 0.00 & 0.00 & 0.00 & -0.01 & 0.00 \\
\hline 0.00 & 0.00 & 0.00 & 0.71 & 0.02 & -0.00 & 0.01 & 0.02 & -0.01 & 0.00 \\
\hline 0.00 & 0.02 & 0.00 & 0.04 & 0.00 & 0.00 & -0.00 & 0.00 & 0.29 & 0.00 \\
\hline 0.00 & 0.17 & 0.00 & 0.01 & -0.00 & 0.13 & 0.00 & -0.00 & 0.00 & 0.00 \\
\hline 0.00 & 0.00 & -0.00 & 2.85 & -0.00 & 0.00 & 0.00 & -0.00 & -0.04 & -0.00 \\
\hline 0.00 & 0.00 & -0.00 & 1.12 & -0.00 & -0.00 & -0.00 & 0.01 & 3.51 & 0.00 \\
\hline 0.00 & 0.00 & 0.00 & 0.45 & 0.00 & 0.02 & 0.00 & -0.00 & 0.08 & -0.00 \\
\hline 0.00 & 0.00 & 0.00 & 1.30 & -0.00 & -0.00 & 0.00 & -0.00 & -0.01 & -0.00 \\
\hline 0.00 & -0.00 & 0.00 & 1.62 & 0.00 & 0.08 & -0.00 & 0.00 & -0.02 & 0.15 \\
\hline-0.00 & 0.00 & 0.00 & 1.46 & 0.00 & 0.01 & -0.00 & 0.19 & -0.00 & 0.00 \\
\hline 0.00 & 0.00 & 0.01 & 0.61 & 0.00 & 0.05 & -0.00 & 0.00 & -0.00 & 0.01 \\
\hline-0.00 & 0.00 & -0.00 & 0.75 & 0.04 & -0.00 & 0.00 & 0.00 & 0.24 & -0.00 \\
\hline 0.00 & 0.00 & 0.00 & 1.01 & -0.00 & 0.00 & 0.12 & -0.00 & -0.01 & 0.00 \\
\hline-0.00 & -0.00 & -0.00 & 4.91 & -0.00 & -0.00 & -0.00 & -0.01 & -0.04 & -0.00 \\
\hline 0.00 & 0.00 & -0.00 & 9.22 & -0.00 & -0.01 & 0.00 & -0.01 & -0.26 & 0.00 \\
\hline-0.00 & 0.00 & 0.00 & 1.50 & 0.01 & -0.00 & -0.00 & 0.01 & -0.02 & -0.00 \\
\hline 0.00 & -0.00 & 0.01 & 0.20 & 0.04 & 0.00 & 0.00 & 0.00 & 0.03 & -0.00 \\
\hline 0.00 & -0.00 & -0.00 & 0.70 & 0.00 & 0.00 & 0.00 & 0.29 & -0.00 & 0.00 \\
\hline-0.00 & 0.00 & 0.00 & 2.90 & 0.00 & -0.00 & 0.00 & 0.08 & -0.01 & -0.00 \\
\hline-0.00 & -0.00 & -0.00 & 8.67 & -0.00 & 0.00 & -0.00 & -0.02 & -0.17 & -0.00 \\
\hline 0.00 & 0.00 & 0.00 & 0.60 & -0.00 & 0.00 & 0.00 & -0.00 & 0.06 & 0.00 \\
\hline 0.00 & 0.00 & -0.00 & 1.74 & -0.00 & -0.00 & 0.00 & 0.00 & -0.01 & 0.00 \\
\hline 0.02 & 0.00 & 0.01 & 0.07 & 0.00 & -0.00 & 0.06 & 0.00 & 0.00 & 0.00 \\
\hline 0.09 & 0.00 & 0.00 & 0.00 & 0.00 & 0.00 & 0.00 & 0.01 & 0.00 & 0.00 \\
\hline-0.00 & -0.00 & -0.00 & 4.64 & -0.00 & 0.00 & 0.00 & 0.00 & 0.64 & 0.00 \\
\hline 0.00 & -0.00 & -0.00 & 3.62 & -0.00 & -0.00 & -0.00 & -0.01 & -0.06 & -0.00 \\
\hline 0.00 & -0.00 & -0.00 & 0.51 & 0.00 & 0.00 & -0.00 & 0.00 & 0.66 & 0.00 \\
\hline 0.00 & 0.00 & 0.03 & 0.03 & 0.00 & 0.20 & -0.00 & 0.00 & -0.00 & -0.00 \\
\hline-0.00 & 0.00 & 0.00 & 1.48 & 0.00 & 0.04 & 0.00 & -0.00 & -0.03 & 0.00 \\
\hline 0.00 & -0.00 & -0.00 & 25.18 & -0.00 & -0.00 & 0.00 & -0.12 & -0.83 & -0.00 \\
\hline 0.00 & 0.00 & 0.00 & 2.35 & -0.00 & 0.15 & -0.00 & -0.00 & -0.01 & 0.00 \\
\hline-0.00 & 0.00 & 0.00 & 2.57 & 0.00 & 0.08 & -0.00 & -0.00 & -0.04 & 0.00 \\
\hline 0.00 & 0.00 & 0.00 & 1.02 & 0.00 & -0.00 & 0.00 & 0.02 & 0.03 & 0.00 \\
\hline-0.00 & 0.00 & 0.00 & 0.98 & 0.00 & 0.02 & 0.00 & 0.00 & 0.05 & 0.01 \\
\hline 0.00 & 0.00 & -0.00 & 3.37 & 0.00 & -0.00 & 0.00 & 0.00 & 0.55 & -0.00 \\
\hline-0.00 & 0.00 & 0.00 & 3.00 & 0.00 & -0.00 & 0.00 & 0.07 & 0.00 & -0.00 \\
\hline 0.00 & -0.00 & 0.00 & 0.04 & 0.00 & 0.44 & -0.00 & 0.01 & 0.00 & 0.55 \\
\hline 0.00 & 0.00 & -0.00 & 0.95 & -0.00 & -0.00 & 0.02 & 0.00 & 0.09 & 0.00 \\
\hline 0.00 & -0.00 & 0.00 & 0.30 & -0.00 & -0.00 & 0.00 & 0.00 & 0.18 & 0.00 \\
\hline-0.00 & -0.00 & 0.00 & 1.73 & 0.04 & 0.00 & -0.00 & 0.00 & 0.58 & $-0.00)$ \\
\hline 0.00 & -0.00 & 0.02 & 0.00 & 0.01 & 0.00 & -0.00 & 0.02 & 0.21 & $0.00)$ \\
\hline
\end{tabular}


Table 6. The $\psi_{4}$ response to $\{1\}, \ldots\{9\},\{0\}$

\begin{tabular}{|c|c|c|c|c|c|c|c|c|c|}
\hline$\{1\}$ & $\{2\}$ & $\{3\}$ & $\{4\}$ & $\{5\}$ & $\{6\}$ & $\{7\}$ & $\{8\}$ & $\{9\}$ & $\{0\}$ \\
\hline 0.00 & 0.00 & 0.00 & 1.60 & 0.00 & -0.00 & 0.00 & -0.01 & 0.23 & -0.00 \\
\hline-0.00 & 0.00 & -0.00 & 0.44 & 0.00 & 0.01 & 0.03 & 0.10 & 0.00 & 0.66 \\
\hline 0.00 & 0.00 & 0.00 & 1.09 & -0.00 & -0.00 & 0.00 & -0.00 & -0.03 & 0.00 \\
\hline 0.00 & -0.00 & 0.00 & 0.08 & -0.00 & 0.00 & -0.00 & 0.00 & -0.00 & -0.00 \\
\hline-0.00 & -0.00 & 0.00 & 0.49 & 0.00 & 0.00 & -0.00 & 0.00 & -0.02 & 0.01 \\
\hline-0.00 & 0.32 & 0.00 & 0.33 & 0.00 & 0.20 & -0.00 & 0.11 & -0.01 & 0.02 \\
\hline-0.00 & 0.00 & -0.00 & 0.08 & 0.00 & 0.00 & 0.00 & 0.02 & 0.00 & -0.00 \\
\hline-0.00 & 0.01 & -0.00 & 16.56 & 0.01 & -0.00 & 0.03 & -0.00 & 0.35 & 0.00 \\
\hline 0.00 & 0.00 & 0.00 & 0.08 & 0.00 & -0.00 & 0.00 & -0.00 & 0.09 & -0.00 \\
\hline-0.00 & 0.00 & -0.00 & 0.75 & 0.00 & -0.03 & 0.00 & 0.00 & 0.15 & 0.00 \\
\hline 0.00 & 0.00 & 0.00 & 0.71 & -0.00 & 0.00 & -0.00 & -0.00 & 0.01 & 0.00 \\
\hline-0.00 & 0.29 & 0.01 & 0.04 & 0.00 & -0.00 & 0.00 & -0.00 & 0.00 & 0.00 \\
\hline 0.00 & 0.00 & 0.00 & 0.01 & 0.00 & 0.00 & -0.00 & 0.00 & 0.00 & 0.01 \\
\hline 0.00 & -0.00 & -0.00 & 1.85 & -0.00 & 0.00 & 0.01 & -0.00 & 0.00 & 0.00 \\
\hline 0.00 & -0.00 & -0.00 & 1.12 & -0.00 & 0.00 & 0.00 & 0.00 & -0.01 & 0.00 \\
\hline 0.00 & 0.00 & 0.00 & 0.45 & 0.12 & 0.33 & 0.00 & 0.51 & 0.10 & 0.01 \\
\hline-0.00 & -0.00 & 0.00 & 1.30 & 0.00 & -0.00 & -0.01 & -0.00 & -0.14 & 0.00 \\
\hline-0.00 & 0.00 & 0.00 & 1.62 & -0.00 & -0.00 & 0.00 & 0.01 & -0.00 & -0.00 \\
\hline-0.00 & 0.00 & -0.00 & 1.46 & 0.00 & -0.00 & 0.00 & 0.00 & 0.00 & 0.00 \\
\hline 0.00 & 0.00 & 0.00 & 0.61 & 0.00 & -0.00 & -0.00 & -0.00 & 0.54 & -0.00 \\
\hline-0.00 & -0.00 & 0.00 & 0.75 & -0.00 & -0.00 & 0.00 & 0.00 & -0.20 & 0.00 \\
\hline-0.00 & 0.00 & 0.00 & 1.01 & 0.00 & 0.51 & -0.00 & 0.00 & 0.00 & -0.00 \\
\hline-0.00 & 0.00 & -0.00 & 4.91 & 0.00 & -0.00 & -0.00 & 0.00 & -0.01 & -0.00 \\
\hline-0.00 & 0.00 & 0.00 & 9.22 & -0.00 & -0.00 & 0.00 & -0.00 & -0.08 & 0.00 \\
\hline-0.00 & 0.00 & -0.00 & 1.50 & 0.00 & 0.04 & 0.26 & 0.00 & 0.32 & 0.05 \\
\hline 0.00 & 0.00 & 0.12 & 0.20 & 0.14 & 0.88 & 0.01 & 1.06 & 0.43 & 0.14 \\
\hline 0.00 & -0.00 & 0.00 & 0.70 & 0.01 & 0.00 & 0.00 & 0.00 & -0.01 & 0.01 \\
\hline-0.00 & -0.00 & 0.00 & 2.90 & -0.00 & -0.00 & -0.00 & -0.00 & -0.05 & -0.00 \\
\hline-0.00 & -0.00 & 0.00 & 8.67 & -0.00 & -0.00 & 0.00 & -0.00 & 0.00 & 0.00 \\
\hline-0.00 & -0.00 & 0.00 & 0.60 & -0.00 & 0.00 & 0.01 & 0.09 & 1.06 & -0.00 \\
\hline 0.00 & -0.00 & 0.00 & 1.74 & -0.00 & -0.00 & 0.00 & -0.00 & -0.10 & 0.00 \\
\hline-0.00 & 0.00 & -0.00 & 0.07 & -0.00 & 0.01 & 0.05 & -0.00 & 0.42 & 0.06 \\
\hline-0.00 & -0.00 & 0.00 & 0.00 & 0.00 & 0.08 & 0.01 & 0.00 & -0.05 & -0.00 \\
\hline-0.00 & 0.03 & -0.00 & 4.64 & -0.00 & 0.00 & 0.02 & -0.00 & 0.00 & -0.00 \\
\hline-0.00 & -0.00 & 0.00 & 3.62 & -0.00 & -0.00 & -0.00 & -0.01 & 0.73 & 0.00 \\
\hline 0.00 & 0.00 & 0.00 & 0.51 & 0.00 & -0.00 & -0.00 & -0.00 & -0.00 & 0.43 \\
\hline-0.00 & -0.00 & -0.00 & 0.03 & 0.00 & -0.00 & -0.00 & 0.00 & 0.00 & -0.00 \\
\hline 0.00 & 0.00 & 0.00 & 1.48 & 0.00 & 0.02 & 0.00 & 0.00 & -0.02 & 0.00 \\
\hline-0.00 & 0.00 & -0.00 & 25.18 & -0.00 & -0.03 & 0.00 & -0.01 & 0.00 & -0.00 \\
\hline 0.00 & -0.00 & -0.00 & 2.35 & 0.00 & -0.00 & 0.00 & -0.00 & -0.00 & -0.00 \\
\hline 0.00 & 0.00 & -0.00 & 2.57 & 0.00 & -0.00 & -0.00 & -0.00 & -0.05 & -0.00 \\
\hline 0.00 & 0.01 & 0.01 & 1.02 & -0.00 & 0.00 & 0.04 & 0.01 & 0.69 & 0.05 \\
\hline 0.00 & -0.00 & 0.00 & 0.98 & 0.00 & 0.00 & 0.00 & 0.02 & -0.01 & -0.00 \\
\hline-0.00 & 0.00 & 0.00 & 3.37 & -0.00 & -0.01 & 0.04 & -0.00 & 0.31 & 0.00 \\
\hline-0.00 & 0.00 & 0.00 & 3.00 & -0.00 & 0.00 & 0.00 & -0.01 & -0.00 & 0.00 \\
\hline 0.00 & 0.00 & 0.00 & 0.04 & 0.00 & -0.00 & -0.00 & -0.00 & -0.03 & -0.00 \\
\hline-0.00 & 0.00 & -0.00 & 0.95 & -0.00 & -0.00 & 0.00 & 0.01 & 0.74 & 0.00 \\
\hline 0.01 & 0.00 & 0.00 & 1.30 & 0.00 & -0.01 & -0.00 & -0.00 & 0.82 & 0.06 \\
\hline 0.01 & 0.02 & 0.00 & 1.73 & -0.01 & 0.02 & -0.00 & 0.01 & 0.90 & 0.11 \\
\hline-0.00 & -0.00 & -0.00 & 0.00 & 0.00 & 0.00 & -0.00 & 0.00 & -0.09 & 0.00 \\
\hline
\end{tabular}


Table 7. The $\varphi_{i}$ responses to the numeral 4

\begin{tabular}{|c|c|c|c|c|c|c|c|c|c|}
\hline$\varphi_{1}$ & $\varphi_{2}$ & $\varphi_{3}$ & $\varphi_{4}$ & $\varphi_{s}$ & $\varphi_{6}$ & $\rho_{7}$ & $\varphi_{8}$ & $\varphi_{9}$ & $\left.p_{0}\right)$ \\
\hline-0.11 & 0.23 & -0.53 & 0.48 & 0.84 & -0.05 & -0.01 & 0.02 & 0.71 & 0.20 \\
\hline-0.04 & 0.61 & -0.29 & 0.59 & 0.57 & 0.47 & 0.09 & -1.16 & 0.52 & -0.38 \\
\hline-0.52 & -0.67 & -0.01 & 0.67 & 0.20 & 0.24 & -0.15 & 0.46 & 0.42 & -0.16 \\
\hline-0.05 & 0.04 & 0.56 & 0.69 & 0.17 & -0.41 & -0.24 & -0.44 & 0.45 & -0.15 \\
\hline-0.19 & 0.53 & -0.93 & 0.74 & -0.49 & -0.12 & 0.45 & 0.30 & 0.13 & 0.02 \\
\hline 0.11 & -0.19 & -1.08 & 0.06 & 0.21 & 0.13 & 0.26 & 0.93 & 0.61 & -0.33 \\
\hline-0.32 & -0.47 & 0.05 & 0.51 & 0.01 & 0.21 & 0.52 & 0.31 & -0.06 & -0.42 \\
\hline-0.51 & 0.28 & -0.21 & 1.72 & 0.23 & -0.34 & 0.02 & -0.20 & -0.06 & -0.19 \\
\hline-1.07 & 0.51 & 0.48 & 1.01 & -0.47 & 0.69 & 0.61 & 0.16 & -0.96 & -0.49 \\
\hline-0.32 & 0.50 & 0.53 & 1.46 & -0.34 & -0.26 & -0.55 & -0.22 & -0.18 & 0.24 \\
\hline 0.32 & -0.36 & -0.16 & 1.15 & 0.33 & -0.53 & 0.34 & 0.69 & -0.59 & -0.21 \\
\hline-0.08 & 0.48 & -0.57 & 0.08 & -0.08 & -0.15 & -0.18 & 0.61 & 0.89 & -0.53 \\
\hline-0.06 & 0.66 & 0.22 & 0.35 & -0.39 & 0.66 & -0.17 & -0.69 & 0.37 & -0.04 \\
\hline 0.02 & 0.20 & -0.70 & 0.76 & -0.28 & 0.46 & 0.23 & 0.23 & 0.19 & 0.13 \\
\hline-0.44 & 0.62 & -0.46 & 1.03 & -0.43 & -0.71 & 0.13 & 0.12 & 0.51 & (0.59 \\
\hline 0.81 & 0.01 & -0.08 & 0.44 & 0.34 & 0.41 & -0.42 & -0.97 & 0.96 & 0.06 \\
\hline 0.50 & -0.09 & 0.44 & 1.77 & -0.02 & -0.48 & 0.02 & -0.42 & -0.74 & 0.23 \\
\hline 0.56 & -0.11 & -0.78 & 0.94 & 0.27 & 0.32 & -0.27 & -0.82 & 0.22 & 1.02 \\
\hline 0.78 & 0.19 & -0.59 & 1.37 & -0.65 & -0.07 & 0.11 & 0.57 & -0.91 & 0.89 \\
\hline-0.31 & -0.08 & 1.12 & 1.94 & -0.26 & -0.06 & -0.01 & -0.28 & -1.38 & 0.59 \\
\hline-0.64 & -0.64 & 0.31 & 0.69 & 0.27 & 0.10 & -0.10 & 0.43 & 0.47 & -0.54 \\
\hline 0.23 & 0.42 & -0.44 & 1.45 & 0.04 & -0.75 & 0.51 & 0.19 & -0.68 & $0 .(0)$ \\
\hline 0.43 & -0.20 & 0.27 & 1.80 & 0.03 & 0.02 & 0.11 & -0.48 & -0.78 & 0.31 \\
\hline 0.28 & 0.25 & -0.09 & 1.79 & -0.20 & -0.24 & -0.08 & -0.63 & -0.31 & 0.86 \\
\hline-0.09 & -0.29 & 0.19 & 0.99 & 0.46 & 0.14 & 0.27 & 0.15 & -0.15 & -0.63 \\
\hline-0.54 & -0.53 & 0.89 & 0.81 & 0.30 & 0.21 & 0.22 & 0.06 & -0.00 & -0.74 \\
\hline 0.31 & -0.01 & -0.11 & 0.86 & 0.27 & 0.06 & 0.66 & 0.34 & -0.50 & -0.41 \\
\hline 0.09 & 0.18 & -0.75 & 0.79 & 0.16 & 0.03 & 0.20 & 0.63 & -0.17 & -0.4 .3 \\
\hline-0.34 & 0,21 & -0.36 & 1.30 & 0.07 & -0.47 & -0.59 & 0.62 & 0.47 & -0.24 \\
\hline 0.34 & -0.39 & 0.47 & 0.96 & -0.29 & 0.20 & 0.15 & -0.11 & 0.03 & -0.25 \\
\hline 0.18 & -0.86 & 0.39 & 1.30 & 0.07 & -0.11 & -0.23 & -0.75 & 0.26 & 0.17 \\
\hline 0.07 & -0.23 & 0.66 & 1.21 & -0.02 & -0.47 & 0.54 & 0.22 & -0.79 & -0.44 \\
\hline 0.36 & -0.19 & -0.32 & 0.09 & -0.25 & -0.06 & 0.10 & 0.99 & -0.05 & -0.19 \\
\hline-0.52 & 0.32 & 0.02 & 1.34 & 0.43 & -0.68 & -0.49 & -0.15 & 0.55 & 0.02 \\
\hline 0.78 & -0.30 & 0.50 & 1.65 & -0.27 & 0.13 & 0.06 & -0.51 & -0.50 & 0.09 \\
\hline-0.21 & -0.28 & -0.39 & 0.15 & 0.25 & 0.66 & 0.15 & -0.51 & 1.07 & -0.08 \\
\hline 0.34 & -0.50 & 0.74 & 0.61 & -0.17 & 0.55 & -0.49 & 0.06 & 0.04 & -0.27 \\
\hline 0.14 & 0.60 & 0.22 & 1.39 & -0.31 & 0.20 & -0.16 & -1.13 & -0.09 & 0.67 \\
\hline 0.27 & -0.01 & -0.41 & 1.71 & 0.06 & -0.25 & -0.07 & 0.26 & -0.15 & -0.17 \\
\hline 0.00 & -0.09 & 0.43 & 1.63 & -0.11 & 0.45 & 0.23 & -0.56 & -0.91 & 0.32 \\
\hline 0.25 & -0.55 & 0.88 & 1.52 & -0.49 & 0.23 & -0.65 & -0.11 & -0.07 & $0.1,3$ \\
\hline-0.51 & -0.14 & 0.18 & 0.94 & 0.13 & -0.04 & 0.00 & 0.61 & 0.04 & -0.74 \\
\hline 0.13 & 0.32 & 0.40 & 1.11 & -0.29 & 0.00 & -0.74 & -0.75 & 0.53 & 0.79 \\
\hline-0.11 & 0.60 & -1.16 & 0.58 & 0.24 & -0.02 & -0.12 & 0.22 & 0.91 & -0.38 \\
\hline 0.03 & 0.30 & 0.63 & 2.37 & 0.33 & -0.71 & -0.30 & 0.02 & -1.57 & 0.30 \\
\hline-0.54 & 0.37 & 0.16 & 0.98 & -0.31 & 0.44 & 0.05 & 0.05 & -0.87 & 0.95 \\
\hline 0.10 & 0.48 & -1.30 & 0.48 & -0.11 & 0.07 & 0.50 & 0.51 & 0.25 & -0.10 \\
\hline 0.78 & -0.73 & 0.18 & 0.84 & -0.23 & 0.06 & -0.19 & 0.07 & 0.44 & 0.22 \\
\hline-0.35 & -0.70 & 0.12 & 0.85 & 0.48 & -0.15 & 0.05 & 0.27 & 0.42 & -0.23 \\
\hline-0.34 & -0.91 & 0.70 & 0.06 & -0.30 & 0.01 & -0.34 & 0.82 & 0.72 & -0.05 \\
\hline
\end{tabular}


Table 8. The $\varphi_{4}$ response to $\{1\}, \ldots\{9\},\{0\}$

\begin{tabular}{|c|c|c|c|c|c|c|c|c|c|}
\hline$\{1\}$ & $\{2\}$ & $\{3\}$ & $\{4\}$ & $\{5\}$ & $\{6\}$ & $\{7\}$ & $\{8\}$ & $\{9\}$ & $\{0\}$ \\
\hline 0.25 & 0.27 & -0.85 & 0.48 & -0.02 & 0.12 & -0.63 & 0.56 & 0.03 & -0.35 \\
\hline-0.11 & 0.40 & -0.29 & 0.59 & 0.64 & 0.41 & 0.25 & 0.11 & 0.03 & 0.75 \\
\hline 0.64 & 0.10 & 0.86 & 0.67 & -0.01 & 0.00 & 0.65 & -0.97 & -0.27 & -0.30 \\
\hline-0.21 & 0.04 & 0.69 & 0.69 & -0.39 & -0.05 & 0.40 & 0.09 & -0.18 & -0.46 \\
\hline 0.23 & -0.44 & -0.13 & 0.74 & 0.53 & 0.00 & 0.06 & -0.44 & -0.08 & 0.24 \\
\hline 0.20 & 1.11 & 0.25 & 0.06 & 0.25 & 0.76 & -0.36 & 0.34 & -0.08 & 0.71 \\
\hline-0.38 & 0.31 & -0.16 & 0.51 & 0.12 & -0.32 & 0.29 & 0.33 & 0.68 & -0.03 \\
\hline-0.36 & 0.97 & 0.12 & 1.72 & 0.19 & -0.63 & 1.07 & 0.49 & 0.33 & 0.25 \\
\hline-0.31 & 0.54 & 0.00 & 1.01 & -0.61 & 0.39 & 0.08 & -0.24 & 0.45 & 0.24 \\
\hline-0.70 & 0.16 & 0.10 & 1.46 & -0.40 & 0.11 & 0.63 & 0.39 & 0.31 & -0.14 \\
\hline 0.67 & 0.36 & -0.35 & 1.15 & 0.36 & 0.52 & -0.67 & -0.20 & 0.50 & 0.62 \\
\hline-0.16 & 1.21 & 0.49 & 0.08 & 0.07 & -0.04 & 0.14 & 0.12 & -0.25 & 0.45 \\
\hline-0.19 & 0.09 & 0.02 & 0.35 & -0.14 & 0.22 & -0.05 & -0.29 & -0.14 & 0.57 \\
\hline 0.55 & -0.81 & 0.04 & 0.76 & 0.40 & -0.45 & -0.63 & 0.35 & -0.29 & 0.46 \\
\hline-0.38 & -1.03 & 0.09 & 1.03 & -0.20 & 0.04 & -0.75 & -0.39 & -0.53 & -0.23 \\
\hline 0.25 & -0.28 & -0.66 & 0.44 & 0.44 & 0.46 & 0.36 & 0.79 & 0.36 & 0.82 \\
\hline-0.15 & 0.11 & 0.27 & 1.77 & 0.17 & 0.09 & 0.32 & -0.91 & -0.53 & -0.39 \\
\hline 0.23 & -0.28 & 0.60 & 0.94 & -0.73 & -0.62 & -1.14 & 0.10 & -0.81 & -0.20 \\
\hline-0.01 & -0.39 & -0.36 & 1.37 & 0.00 & 0.17 & -0.36 & -0.56 & -0.56 & -0.09 \\
\hline 0.02 & -1.56 & 0.29 & 1.94 & 0.37 & -0.29 & 0.21 & 0.20 & 0.45 & -1.10 \\
\hline 0.06 & 0.83 & -0.17 & 0.69 & 0.15 & -0.56 & -0.44 & 0.25 & -0.20 & -0.65 \\
\hline 0.25 & 0.23 & -0.44 & 1.45 & 0.60 & 0.71 & -0.18 & -0.78 & 0.58 & -0.16 \\
\hline-0.15 & -0.59 & 0.06 & 1.80 & 0.57 & -0.18 & 0.18 & -0.58 & -0.33 & -1.00 \\
\hline 0.46 & -0.24 & -0.02 & 1.79 & -0.40 & -0.34 & -0.76 & 0.54 & -0.35 & -0.45 \\
\hline-0.59 & -0.19 & -0.08 & 0.99 & 0.10 & 0.28 & 1.19 & 0.38 & 0.83 & 0.73 \\
\hline 0.41 & 0.21 & 0.70 & 0.81 & 0.58 & 0.60 & 0.42 & 0.61 & 0.94 & 0.72 \\
\hline 0.44 & -0.66 & -0.21 & 0.86 & 0.28 & -0.26 & -0.62 & 0.26 & -0.41 & 0.62 \\
\hline-0.13 & -0.12 & 0.51 & 0.79 & -0.13 & 0.03 & -0.28 & -0.18 & -0.58 & 0.13 \\
\hline 0.30 & 0.33 & -0.11 & 1.30 & -0.98 & -0.55 & -0.61 & 0.08 & 0.25 & 0.42 \\
\hline 0.10 & 0.01 & -0.94 & 0.96 & 0.26 & -0.40 & -0.69 & 0.65 & 0.77 & -0.27 \\
\hline-0.35 & 0.37 & -0.38 & 1.30 & 0.19 & -0.00 & 0.22 & -0.61 & -0.37 & -0.72 \\
\hline-0.66 & -0.03 & -0.25 & 1.21 & 0.60 & 0.41 & 0.82 & 0.53 & 0.59 & 1.19 \\
\hline-0.43 & -0.56 & -0.22 & 0.09 & 0.09 & 0.23 & 0.45 & -0.75 & 0.39 & 0.97 \\
\hline-0.84 & 0.62 & -0.52 & 1.34 & -0.11 & 0.41 & -0.06 & -0.61 & -0.71 & -0.12 \\
\hline-0.03 & 0.11 & 0.28 & 1.65 & -0.33 & 0.08 & 0.31 & 0.28 & 0.60 & 0.24 \\
\hline 0.25 & -0.04 & 0.56 & 0.15 & 0.35 & -0.40 & 0.62 & 0.29 & -0.43 & 0.95 \\
\hline-0.34 & 0.05 & 0.49 & 0.61 & -0.67 & -0.35 & 0.27 & -0.18 & 0.25 & -0.09 \\
\hline 0.08 & 0.23 & -0.05 & 1.39 & -0.43 & 0.10 & 0.05 & -0.33 & -0.71 & 0.72 \\
\hline-0.20 & -0.51 & 0.20 & 1.71 & 0.28 & 0.10 & -0.70 & 0.08 & -0.42 & -0.36 \\
\hline-0.40 & -0.21 & 0.12 & 1.63 & -0.34 & -0.15 & 0.12 & -0.55 & -0.11 & -0.17 \\
\hline 0.17 & -0.73 & 0.15 & 1.52 & -0.46 & -0.01 & -0.18 & 0.37 & -0.27 & -0.49 \\
\hline 0.46 & 0.27 & 0.20 & 0.94 & -0.13 & 0.13 & 0.87 & 0.10 & 0.54 & 0.57 \\
\hline-0.48 & -0.48 & 0.19 & 1.11 & -0.82 & -0.29 & -0.25 & -0.53 & -0.30 & -1.56 \\
\hline 0.41 & -0.01 & -0.33 & 0.58 & 0.13 & -0.10 & 0.67 & -1.14 & 0.45 & 0.55 \\
\hline-0.64 & 0.17 & -0.78 & 2.37 & -0.37 & 0.87 & -0.98 & 0.37 & -0.40 & -0.78 \\
\hline 0.38 & -0.86 & -0.33 & 0.98 & -0.54 & -0.86 & 0.14 & 0.26 & -0.49 & -1.44 \\
\hline-0.15 & -0.14 & -0.08 & 0.48 & 0.10 & -0.23 & -0.47 & 0.74 & 0.56 & -0.11 \\
\hline 0.97 & 0.27 & -0.23 & 0.84 & 0.53 & -0.05 & -0.03 & 0.21 & -0.42 & 0.01 \\
\hline 0.79 & 0.17 & 0.51 & 0.85 & 0.06 & 0.49 & 0.07 & 0.69 & 0.17 & 0.73 \\
\hline-0.23 & 0.60 & -0.66 & 0.06 & -0.18 & -0.75 & 0.01 & 0.71 & -0.67 & -1.01 \\
\hline
\end{tabular}


Table 9. The $\bar{p}_{k}$ responses to the numeral 4

\begin{tabular}{|c|c|c|c|c|c|c|c|c|c|}
\hline $\bar{p}_{1}$ & $\bar{p}_{2}$ & $\bar{p}_{3}$ & $\bar{p}_{4}$ & $\bar{p}_{5}$ & $\bar{p}_{6}$ & $\bar{p}_{7}$ & $\bar{p}_{8}$ & $\bar{p}_{9}$ & $\bar{p}_{0}$ \\
\hline 8.79 & 13.69 & 6.75 & 7.78 & 5.54 & 10.02 & 7.59 & 7.03 & 3.25 & 11.77 \\
\hline 9.82 & 5.65 & 14.47 & 2.51 & 4.89 & 5.93 & 7.52 & 3.17 & 6.04 & 2.31 \\
\hline 7.89 & 8.45 & 8.15 & 2.76 & 6.43 & 5.12 & 5.65 & 8.08 & 5.44 & 12.22 \\
\hline 9.09 & 8.41 & 6.09 & 3.16 & 7.13 & 5.26 & 6.83 & 5.18 & 8.91 & 8.54 \\
\hline 9.68 & 11.53 & 10.38 & 4.56 & 5.18 & 5.59 & 7.08 & 7.38 & 4.45 & 4.07 \\
\hline 9.74 & 3.87 & 7.26 & 3.34 & 5.59 & 3.26 & 8.61 & 2.48 & 4.87 & 4.67 \\
\hline 10.27 & 9.19 & 9.09 & 3.39 & 7.01 & 8.30 & 9.78 & 4.75 & 6.24 & 10.00 \\
\hline 11.24 & 6.19 & 10.33 & 2.07 & 4.85 & 8.79 & 4.51 & 6.03 & 2.27 & 11.75 \\
\hline 8.43 & 7.51 & 11.49 & 4.70 & 11.13 & 6.30 & 5.85 & 7.37 & 3.14 & 13.09 \\
\hline 10.24 & 6.15 & 7.48 & 2.64 & 8.73 & 7.92 & 7.00 & 7.87 & 3.75 & 12.33 \\
\hline 8.58 & 5.68 & 8.35 & 2.74 & 7.75 & 6.85 & 9.81 & 4.33 & 4.68 & 5.75 \\
\hline 11.18 & 3.48 & 3.08 & 4.06 & 5.00 & 5.73 & 5.14 & 7.91 & 4.54 & 8.26 \\
\hline 7.25 & 6.24 & 5.05 & 4.80 & 8.61 & 4.80 & 5.88 & 6.05 & 3.98 & 5.53 \\
\hline 6.75 & 12.70 & 11.03 & 1.88 & 11,90 & 11.73 & 12.08 & 11.83 & 4.69 & 11.47 \\
\hline 7.01 & 11.73 & 13.02 & 5.13 & 8.55 & 12.18 & 10.13 & 8.12 & 6.29 & 10.25 \\
\hline 6.60 & 5.23 & 6.67 & 2.48 & 1.59 & 2.81 & 6.82 & 1.83 & 2.31 & 6.48 \\
\hline 10.43 & 9.52 & 10.36 & 1.97 & 9.94 & 7.62 & 7.63 & 5.00 & 8.51 & 13.61 \\
\hline 8.80 & 16.77 & 8.31 & 5.06 & 9.24 & 9.53 & 14.31 & 3.80 & 5.42 & 13.79 \\
\hline 9.02 & 15.28 & 11.73 & 5.46 & 7.74 & 9.13 & 13.59 & 9.70 & 10.46 & 12.31 \\
\hline 9.22 & 12.53 & 10.65 & 5.30 & 5.73 & 11.35 & 5.78 & 11.16 & 3.18 & 16.88 \\
\hline 9.83 & 8.97 & 9.45 & 3.01 & 9.08 & 8.39 & 9.57 & 5.15 & 5.96 & 13.85 \\
\hline 10.32 & 6.22 & 8.62 & 3.37 & 5.37 & 3.36 & 7.19 & 7.10 & 5.40 & 6.54 \\
\hline 11.56 & 6.88 & 7.15 & 2.04 & 4.80 & 5.06 & 7.96 & 9.25 & 8.98 & 14.55 \\
\hline 9.71 & 12.04 & 14.54 & 3.56 & 11.99 & 7.55 & 12.76 & 8.98 & 4.39 & 16.07 \\
\hline 10.04 & 6.08 & 8.68 & 1.61 & 6.97 & 5.43 & 3.95 & 5.38 & 4.43 & 4.94 \\
\hline 7.85 & 5.37 & 4.63 & 2.91 & 4.13 & 2.80 & 4.32 & 1.79 & 2.15 & 5.10 \\
\hline 7.90 & 8.15 & 9.17 & 4.04 & 4.03 & 5.68 & 10.86 & 7.04 & 13.79 & 8.89 \\
\hline 10.29 & 12.26 & 8.88 & 2.20 & 9.93 & 5.62 & 5.61 & 5.37 & 4.51 & 9.87 \\
\hline 9.55 & 5.25 & 7.74 & 3.08 & 10.19 & 8.14 & 10.47 & 6.92 & 4.36 & 7.91 \\
\hline 10.21 & 9.28 & 9.24 & 1.34 & 8.12 & 5.76 & 11.20 & 2.71 & 5.33 & 7.59 \\
\hline 8.59 & 8.82 & 7.81 & 1.73 & 5.77 & 6.82 & 6.45 & 12.7 & 5.38 & 10.88 \\
\hline 11.04 & 7.51 & 6.83 & 4.10 & 6.98 & 4.53 & 4.16 & 8.52 & 2.67 & 6.31 \\
\hline 10.46 & 7.93 & 6.62 & 5.30 & 5.23 & 3.42 & 4.78 & 5.29 & 3.85 & 9.28 \\
\hline 12.56 & 4.75 & 7.11 & 2.95 & 8.98 & 7.05 & 10.76 & 7.05 & 8.70 & 8.69 \\
\hline 10.97 & 7.99 & 7.59 & 3.28 & 10.85 & 7.84 & 7.98 & 7.73 & 2.01 & 9.80 \\
\hline 9.08 & 8.82 & 5.68 & 2.85 & 4.45 & 7.15 & 6.32 & 5.97 & 4.65 & 2.40 \\
\hline 10.57 & 8.46 & 8.67 & 3.92 & 6.47 & 7.85 & 6.26 & 10.65 & 3.80 & 15.72 \\
\hline 6.91 & 6.87 & 13.51 & 3.64 & 10.01 & 4.13 & 9.33 & 6.47 & 7.34 & 7.91 \\
\hline 11.03 & 17.17 & 8.91 & 2.81 & 7.95 & 6.67 & 9.59 & 9.53 & 10.94 & 6.47 \\
\hline 8.59 & 13.07 & 12.28 & 2.81 & 5.58 & 10.03 & 11.30 & 14.59 & 9.49 & 13.22 \\
\hline 5.92 & 10.60 & 12.48 & 2.95 & 5.88 & 6.05 & 9.87 & 10.58 & 8.49 & 16.38 \\
\hline 7.46 & 5.80 & 5.97 & 1.92 & 8.67 & 5.63 & 6.03 & 5.43 & 2.08 & 3.39 \\
\hline 7.35 & 19.11 & 10.11 & 4.43 & 18.42 & 9.06 & 5.51 & 13.75 & 9.96 & 20.98 \\
\hline 8.99 & 5.34 & 6.46 & 3.07 & 8.27 & 7.27 & 4.12 & 11.19 & 3.47 & 3.30 \\
\hline 12.11 & 12.39 & 13.13 & 4.93 & 11.00 & 8.80 & 13.60 & 8.86 & 9.06 & 14.40 \\
\hline 6.82 & 14.35 & 14.42 & 8.14 & 11.97 & 9.57 & 8.00 & 11.28 & 7.58 & 19.11 \\
\hline 10.99 & 6.89 & 6.31 & 3.19 & 7.14 & 6.48 & 10.81 & 6.13 & 2.06 & 15.68 \\
\hline 6.86 & 13.69 & 14.16 & 2.19 & 7.76 & 8.45 & 9.43 & 7.19 & 3.23 & 5.32 \\
\hline 7.80 & 5.54 & 5.90 & 2.72 & 8.84 & 5.07 & 8.85 & 4.59 & 3.74 & 6.84 \\
\hline 9.66 & 13.36 & 9.81 & 4.88 & 12.50 & 8.83 & 5.50 & 7.85 & 8.63 & 12.29 \\
\hline
\end{tabular}


Table 10. The $\bar{p}_{k}$ responses to $\{1\}, \ldots\{0\}$

\begin{tabular}{|c|c|c|c|c|c|c|c|c|c|}
\hline$\{1\}$ & $\{2\}$ & $\{3\}$ & $\{4\}$ & $\{5\}$ & $\{6\}$ & $\{7\}$ & $\{8\}$ & $\{9\}$ & $\{0\}$ \\
\hline 24.90 & 16.55 & 16.45 & 7.78 & 11.41 & 13.55 & 14.81 & 8.82 & 8.06 & 14.76 \\
\hline 7.23 & 5.97 & 7.50 & 2.51 & 5.35 & 4.60 & 5.90 & 7.55 & 4.15 & 9.01 \\
\hline 13.40 & 11.18 & 9.60 & 2.76 & 4.96 & 6.09 & 9.11 & 6.08 & 4.08 & 7.38 \\
\hline 5.21 & 5.06 & 3.76 & 3.16 & 4.44 & 6.94 & 4.48 & 5.89 & 2.81 & 6.98 \\
\hline 9.00 & 7.82 & 11.72 & 4.56 & 10.28 & 9.77 & 5.36 & 9.01 & 5.52 & 9.46 \\
\hline 8.86 & 9.16 & 10.32 & 3.34 & 6.02 & 7.91 & 5.90 & 4.95 & 3.58 & 10.36 \\
\hline 5.87 & 7.60 & 6.74 & 3.39 & 4.55 & 6.32 & 3.58 & 6.59 & 4.11 & 7.41 \\
\hline 15.15 & 11.16 & 12.58 & 2.07 & 8.67 & 9.84 & 9.22 & 8.59 & 5.15 & 11.53 \\
\hline 11.06 & 4.63 & 6.49 & 4.70 & 8.09 & 4.03 & 8.05 & 6.62 & 7.50 & 8.51 \\
\hline 11.88 & 4.97 & 6.15 & 2.64 & 7.07 & 5.29 & 10.51 & 5.20 & 5.04 & 7.38 \\
\hline 7.18 & 8.88 & 8.62 & 2.74 & 5.06 & 9.11 & 5.10 & 4.95 & 4.91 & 10.80 \\
\hline 6.70 & 4.79 & 7.49 & 4.06 & 7.53 & 7.91 & 6.77 & 4.66 & 3.26 & 11.28 \\
\hline 6.50 & 2.64 & 4.45 & 4.30 & 7.54 & 2.59 & 7.85 & 6.14 & 5.14 & 6.96 \\
\hline 13.00 & 8.34 & 10.84 & 1.88 & 8.52 & 5.41 & 8.00 & 5.09 & 3.96 & 8.90 \\
\hline 18.61 & 11.06 & 13.24 & 5.13 & 12.60 & 13,54 & 8.28 & 8.89 & 4.65 & 9.34 \\
\hline 7.44 & 7.38 & 7.08 & 2.48 & 6.12 & 4.49 & 7.68 & 5.93 & 3.44 & 9.50 \\
\hline 6.13 & 7.11 & 6.68 & 1.97 & 6.25 & 7.06 & 5.64 & 6.61 & 5.29 & 8.55 \\
\hline 14.34 & 13.56 & 14.14 & 5.06 & 8.42 & 6.83 & 11.10 & 10.95 & 8.34 & 6.08 \\
\hline 13.42 & 9.96 & 12.89 & 5.46 & 12.10 & 8.29 & 11.41 & 7.06 & 9.34 & 10.28 \\
\hline 16.18 & 9.51 & 8.05 & 5.30 & 8.94 & 6.59 & 12.41 & 7.78 & 9.46 & 7.36 \\
\hline 12.17 & 10.51 & 8.44 & 3.01 & 5.01 & 7.22 & 8.44 & 6.13 & 3.73 & 9.39 \\
\hline 7.45 & 7.67 & 10.01 & 3.37 & 8.18 & 10.94 & 4.25 & 7.34 & 5.65 & 11.03 \\
\hline 10.76 & 9.73 & 9.35 & 2.04 & 7.36 & 6.17 & 8.31 & 7.20 & 6.40 & 9.22 \\
\hline 16.92 & 11.86 & 12.84 & 3.50 & 11.12 & 9.21 & 10.64 & 9.17 & 6.96 & 8.94 \\
\hline 9.45 & 8.38 & 7.52 & 1.61 & 4.04 & 6.22 & 8.56 & 4.45 & 3.73 & 11.38 \\
\hline 8.55 & 7.89 & 5.02 & 2.91 & 4.07 & 6.00 & 6.06 & 5.79 & 3.79 & 9.49 \\
\hline 10.88 & 9.06 & 9.56 & 4.04 & 7.90 & 9.01 & 6.99 & 5.00 & 5.88 & 14.14 \\
\hline 11.04 & 8.62 & 11.03 & 2.20 & 7.64 & 7.97 & 7.79 & 4.60 & 4.08 & 12.94 \\
\hline 15.96 & 10.78 & 12.63 & 3.08 & 9.44 & 10.13 & 12.75 & 6.44 & 5.16 & 13.26 \\
\hline 5.66 & 6.20 & 5.25 & 1.34 & 6.18 & 5.07 & 4.73 & 4.66 & 2.74 & 9.44 \\
\hline 8.53 & 9.10 & 7.52 & 1.73 & 5.88 & 6.42 & 6.28 & 7.81 & 3.65 & 7.19 \\
\hline 4.30 & 6.44 & 5.33 & 4.10 & 6.10 & 9.19 & 3.57 & 6.46 & 5.46 & 10.92 \\
\hline 2.07 & 4.68 & 5.70 & 5.80 & 5.38 & 6.32 & 5.22 & 4.63 & 5.55 & 8.36 \\
\hline 17.07 & 10.80 & 10.98 & 2.95 & 7.32 & 10.52 & 10.06 & 7.11 & 4.11 & 10.33 \\
\hline 11.73 & 10.39 & 9.77 & 2.85 & 5.93 & 5.66 & 6.20 & 7.69 & 2.89 & 7.03 \\
\hline 5.48 & 5.70 & 4.12 & 3.92 & 5.36 & 2.49 & 10.12 & 4.65 & 5.92 & 9.18 \\
\hline 13.76 & 7.78 & 9.07 & 3.64 & 10.20 & 5.65 & 9.91 & 8.44 & 6.35 & 7.38 \\
\hline 14.33 & 12.34 & 14.08 & 2.61 & 10.52 & 10.42 & 11.08 & 7.73 & 6.37 & 15.30 \\
\hline 12.85 & 9.27 & 8.97 & 2.81 & 7.69 & 4.47 & 9.49 & 7.46 & 7.26 & 7.75 \\
\hline 11.95 & 9.41 & 7.83 & 2.95 & 8.91 & 5.08 & 12.70 & 6.47 & 6.19 & 10.33 \\
\hline 9.43 & 7.10 & 7.20 & 1.92 & 5.32 & 6.53 & 7.59 & 4.24 & 6.48 & 11.02 \\
\hline 16.83 & 9.20 & 9.14 & 4.43 & 10.84 & 6.72 & 12.98 & 7.39 & 5.83 & 7.57 \\
\hline 12.43 & 9.24 & 12.02 & 3.07 & 8.99 & 9.20 & 9.02 & 6.99 & 4.15 & 12.93 \\
\hline 15.21 & 10.08 & 10.25 & 4.93 & 8.57 & 9.18 & 14.09 & 7.34 & 10.49 & 11.91 \\
\hline 20.50 & 10.43 & 11.25 & 8.14 & 10.53 & 6.23 & 14.89 & 8.54 & 11.14 & 5.48 \\
\hline 9.43 & 7.84 & 11.94 & 3.19 & 8.62 & 8.44 & 5.34 & 6.31 & 4.35 & 10.45 \\
\hline 9.23 & 9.80 & 8.14 & 2.19 & 7.43 & 6.76 & 7.26 & 5.11 & 3.27 & 9.77 \\
\hline 14.04 & 12.54 & 10.07 & 2.72 & 5.28 & 9.05 & 7.55 & 6.44 & 3.44 & 9.50 \\
\hline 9.56 & 8.14 & 4.72 & 4.88 & 5.00 & 6.53 & 7.64 & 4.53 & 3.25 & 5.94 \\
\hline
\end{tabular}


It was pointed out ${ }^{(2)}$ that pattern recognition functions could be synthesized with specified Frechet derivatives at the test points. In particular, by choosing zero derivatives the pattern recognition function is made very insensitive to small errors. This behavior was observed experimentally. However, is it is a property which the $\psi_{j}$ functions have naturally (see Table 3) and hence experiments in this direction were not pursued.

As a closing remark, we note that the ability of the $\psi_{j}$ functions to illuminate a large number of false choices and order the few possible true choices suggests application where inter-data context can be used.

\section{REFERENCES}

1. W. H. Highleyman. Trans. IRE 10, (1961).

2. W. A. Porter, A class of error tolerant pattern discrimination functions. Pattern Recognition 9. 5964 (1977).

3. J. R. Ullman and P. A. Kidd. Recognition experiments with typed numerals from envelopes in the mail. Pattern Recognition 1. 273-289 (1969).

4. H. C. Andrews. Introduction to mathematical techniques, in Pattern Recognition. Wiley. NY (1972).

\footnotetext{
About the Author-William A. PORTER was born in South Haven, MI. on 11 December. 1934. He attended the Michigan Technological University and subsequently the University of Michigan. Ann Arbor, where he received the doctorate degree in electrical engineering in 1961.

$\mathrm{He}$ is presently Professor of Electrical and Computer Engineering at the University of Michigan, Ann Arbor. His research and teaching interests currently include pattern recognition. neuromuscular systems, non-self-adjoint resolvant operator theory and assorted applications. He has authored two texts and numerous research publications. He is currently also serving as a Consultant with Minneapolis Honeywell in the area of sensitivity reducing design procedures.
} 